\title{
Effects of Tris(pyrazoly l)borato Ligand Substituents on Dioxygen Activation and Stabilization by Copper Compounds
}

\author{
Gabriel Aullón*, Sergiu M. Gorun, Santiago Alvarez
}

\author{
Supporting Information \\ Tables S1 and S2, 20 pages
}

\section{Table of Contents}

Tables S1. Atomic coordinates of optimized structures 1a having short O-O distance:

$$
\begin{array}{ll}
{\left[\mathrm{Cu}_{2}(\square-\mathrm{O})_{2}(\mathrm{Tp})_{2}\right](\mathrm{p} .2)} & {\left[\mathrm{Cu}_{2}(\square-\mathrm{O})_{2}\left(\mathrm{Tp}^{3 F}\right)_{2}\right](\mathrm{p} .3)} \\
{\left[\mathrm{Cu}_{2}(\square-\mathrm{O})_{2}\left(\mathrm{Tp}^{3 C l}\right)_{2}\right](\mathrm{p} .4)} & {\left[\mathrm{Cu}_{2}(\square-\mathrm{O})_{2}\left(\mathrm{Tp}^{3 B r}\right)_{2}\right](\mathrm{p} .5)} \\
{\left[\mathrm{Cu}_{2}(\square-\mathrm{O})_{2}\left(\mathrm{Tp}^{3 I}\right)_{2}\right](\mathrm{p} .6)} & {\left[\mathrm{Cu}_{2}(\square-\mathrm{O})_{2}\left(\mathrm{Tp}^{5 F}\right)_{2}\right](\mathrm{p} .7)} \\
{\left[\mathrm{Cu}_{2}(\square-\mathrm{O})_{2}\left(\mathrm{Tp}^{3 F, 5 F}\right)_{2}\right](\mathrm{p} .8)} & {\left[\mathrm{Cu}_{2}(\square-\mathrm{O})_{2}\left(\mathrm{Tp}^{3 C H_{3}}\right)_{2}\right](\mathrm{p} .9)} \\
{\left[\mathrm{Cu}_{2}(\square-\mathrm{O})_{2}\left(\mathrm{Tp}^{3 C F_{3}}\right)_{2}\right](\mathrm{p} .11)} & {\left[\mathrm{Cu}_{2}(\square-\mathrm{O})_{2}\left(\mathrm{Tp}^{a p-3 C F_{3}}\right)_{2}\right](\mathrm{p} .13)} \\
{\left[\mathrm{Cu}_{2}(\square-\mathrm{O})_{2}\left(\mathrm{Tp}^{b s-3 C F_{3}}\right)_{2}\right](\mathrm{p} .14)} &
\end{array}
$$

Tables S2. Atomic coordinates of optimized structures 1b without interaction across the ring:

$$
\begin{array}{ll}
{\left[\mathrm{Cu}_{2}(\square-\mathrm{O})_{2}(\mathrm{Tp})_{2}\right](\mathrm{p} .16)} & {\left[\mathrm{Cu}_{2}(\square-\mathrm{O})_{2}\left(\mathrm{Tp}^{5 F}\right)_{2}\right](\mathrm{p} .17)} \\
{\left[\mathrm{Cu}_{2}(\square-\mathrm{O})_{2}\left(\mathrm{Tp}^{3 C H_{3}}\right)_{2}\right](\mathrm{p} .18)} & {\left[\mathrm{Cu}_{2}(\square-\mathrm{O})_{2}\left(\mathrm{Tp}^{a p-3 C F_{3}}\right)_{2}\right](\mathrm{p} .20)}
\end{array}
$$


$\mathbf{1 a}-\left[\mathrm{Cu}_{2}(\square-\mathrm{O})_{2}(\mathrm{Tp})_{2}\right]$

\begin{tabular}{|c|c|c|c|}
\hline $\mathrm{Cu}$ & 1.829667 & 0.000000 & 0.000000 \\
\hline $\mathrm{Cu}$ & -1.829667 & 0.000000 & 0.000000 \\
\hline 0 & 0.000000 & 0.705859 & 0.000000 \\
\hline 0 & 0.000000 & -0.705859 & 0.000000 \\
\hline B & 4.839181 & 0.000000 & -0.601162 \\
\hline B & -4.839181 & 0.000000 & 0.601162 \\
\hline $\mathrm{H}$ & 6.024695 & 0.000000 & -0.780231 \\
\hline $\mathrm{H}$ & -6.024695 & 0.000000 & 0.780231 \\
\hline $\mathrm{N}$ & 4.118351 & 0.000000 & -1.971557 \\
\hline $\mathrm{N}$ & 4.417641 & -1.263473 & 0.206739 \\
\hline $\mathrm{N}$ & 4.417641 & 1.263473 & 0.206739 \\
\hline $\mathrm{N}$ & -4.118351 & 0.000000 & 1.971557 \\
\hline $\mathrm{N}$ & -4.417641 & -1.263473 & -0.206739 \\
\hline $\mathrm{N}$ & -4.417641 & 1.263473 & -0.206739 \\
\hline $\mathrm{N}$ & 2.754458 & 0.000000 & -2.057141 \\
\hline $\mathrm{C}$ & 2.453222 & 0.000000 & -3.362960 \\
\hline $\mathrm{C}$ & 3.628951 & 0.000000 & -4.146466 \\
\hline $\mathrm{C}$ & 4.667569 & 0.000000 & -3.225153 \\
\hline $\mathrm{H}$ & 3.706750 & 0.000000 & -5.221401 \\
\hline $\mathrm{H}$ & 5.736340 & 0.000000 & -3.363361 \\
\hline $\mathrm{N}$ & -2.754458 & 0.000000 & 2.057141 \\
\hline $\mathrm{C}$ & -2.453222 & 0.000000 & 3.362960 \\
\hline $\mathrm{C}$ & -3.628951 & 0.000000 & 4.146466 \\
\hline $\mathrm{C}$ & -4.667569 & 0.000000 & 3.225153 \\
\hline $\mathrm{H}$ & -3.706750 & 0.000000 & 5.221401 \\
\hline $\mathrm{H}$ & -5.736340 & 0.000000 & 3.363361 \\
\hline $\mathrm{N}$ & 3.112152 & -1.483135 & 0.533320 \\
\hline $\mathrm{C}$ & 3.056885 & -2.635498 & 1.220449 \\
\hline $\mathrm{C}$ & 4.349945 & -3.181376 & 1.346674 \\
\hline $\mathrm{C}$ & 5.185072 & -2.282316 & 0.690953 \\
\hline $\mathrm{H}$ & 4.632793 & -4.095288 & 1.842275 \\
\hline $\mathrm{H}$ & 6.251211 & -2.292714 & 0.536040 \\
\hline $\mathrm{N}$ & 3.112152 & 1.483135 & 0.533320 \\
\hline $\mathrm{C}$ & 3.056885 & 2.635498 & 1.220449 \\
\hline $\mathrm{C}$ & 4.349945 & 3.181376 & 1.346674 \\
\hline $\mathrm{C}$ & 5.185072 & 2.282316 & 0.690953 \\
\hline $\mathrm{H}$ & 4.632793 & 4.095288 & 1.842275 \\
\hline $\mathrm{H}$ & 6.251211 & 2.292714 & 0.536040 \\
\hline $\mathrm{N}$ & -3.112152 & -1.483135 & -0.533320 \\
\hline $\mathrm{C}$ & -3.056885 & -2.635498 & -1.220449 \\
\hline $\mathrm{C}$ & -4.349945 & -3.181376 & -1.346674 \\
\hline $\mathrm{C}$ & -5.185072 & -2.282316 & -0.690953 \\
\hline $\mathrm{H}$ & -4.632793 & -4.095288 & -1.842275 \\
\hline $\mathrm{H}$ & -6.251211 & -2.292714 & -0.536040 \\
\hline $\mathrm{N}$ & -3.112152 & 1.483135 & -0.533320 \\
\hline $\mathrm{C}$ & -3.056885 & 2.635498 & -1.220449 \\
\hline $\mathrm{C}$ & -4.349945 & 3.181376 & -1.346674 \\
\hline $\mathrm{C}$ & -5.185072 & 2.282316 & -0.690953 \\
\hline $\mathrm{H}$ & -4.632793 & 4.095288 & -1.842275 \\
\hline $\mathrm{H}$ & -6.251211 & 2.292714 & -0.536040 \\
\hline $\mathrm{H}$ & 1.422603 & 0.000000 & -3.684877 \\
\hline $\mathrm{H}$ & -1.422603 & 0.000000 & 3.684877 \\
\hline $\mathrm{H}$ & 2.113448 & -3.011842 & 1.584633 \\
\hline $\mathrm{H}$ & 2.113448 & 3.011842 & 1.584633 \\
\hline $\mathrm{H}$ & -2.113448 & -3.011842 & -1.584633 \\
\hline $\mathrm{H}$ & -2.113448 & 3.011842 & -1.584633 \\
\hline
\end{tabular}




$$
\mathbf{1 a}-\left[\mathrm{Cu}_{2}(\square-\mathrm{O})_{2}\left(\mathrm{Tp}^{3 F}\right)_{2}\right]
$$

\begin{tabular}{|c|c|c|c|}
\hline $\mathrm{Cu}$ & 1.823535 & -0.000167 & -0.000208 \\
\hline $\mathrm{Cu}$ & -1.822967 & -0.000167 & -0.000208 \\
\hline 0 & 0.000283 & 0.701233 & -0.000208 \\
\hline 0 & 0.000283 & -0.701567 & -0.000208 \\
\hline B & 4.818900 & -0.000017 & -0.849483 \\
\hline B & -4.818915 & 0.000000 & 0.849478 \\
\hline $\mathrm{H}$ & 5.986090 & -0.000017 & -1.121900 \\
\hline $\mathrm{H}$ & -5.986105 & 0.000000 & 1.121895 \\
\hline $\mathrm{N}$ & 3.998498 & -0.000017 & -2.159787 \\
\hline $\mathrm{N}$ & 4.461072 & -1.261381 & -0.010590 \\
\hline $\mathrm{N}$ & 4.461072 & 1.261348 & -0.010590 \\
\hline $\mathrm{N}$ & -3.998513 & 0.000000 & 2.159782 \\
\hline $\mathrm{N}$ & -4.461088 & -1.261365 & 0.010585 \\
\hline $\mathrm{N}$ & -4.461088 & 1.261365 & 0.010585 \\
\hline $\mathrm{N}$ & 2.627218 & -0.000017 & -2.128734 \\
\hline $\mathrm{C}$ & 2.251702 & -0.000017 & -3.398789 \\
\hline $\mathrm{C}$ & 3.336240 & -0.000017 & -4.295018 \\
\hline $\mathrm{C}$ & 4.442296 & -0.000017 & -3.455002 \\
\hline $\mathrm{H}$ & 3.307290 & -0.000017 & -5.370304 \\
\hline $\mathrm{H}$ & 5.495567 & -0.000017 & -3.682538 \\
\hline $\mathrm{N}$ & -2.627233 & 0.000000 & 2.128729 \\
\hline $\mathrm{C}$ & -2.251718 & 0.000000 & 3.398785 \\
\hline $\mathrm{C}$ & -3.336256 & 0.000000 & 4.295014 \\
\hline $\mathrm{C}$ & -4.442311 & 0.000000 & 3.454997 \\
\hline $\mathrm{H}$ & -3.307306 & 0.000000 & 5.370299 \\
\hline $\mathrm{H}$ & -5.495582 & 0.000000 & 3.682533 \\
\hline $\mathrm{N}$ & 3.176243 & -1.473783 & 0.416483 \\
\hline $\mathrm{C}$ & 3.202342 & -2.616765 & 1.094875 \\
\hline $\mathrm{C}$ & 4.484241 & -3.184753 & 1.135830 \\
\hline $\mathrm{C}$ & 5.260193 & -2.282563 & 0.414442 \\
\hline $\mathrm{H}$ & 4.785953 & -4.099917 & 1.613465 \\
\hline $\mathrm{H}$ & 6.311099 & -2.297235 & 0.177952 \\
\hline $\mathrm{N}$ & 3.176243 & 1.473750 & 0.416483 \\
\hline $\mathrm{C}$ & 3.202342 & 2.616732 & 1.094875 \\
\hline $\mathrm{C}$ & 4.484241 & 3.184720 & 1.135830 \\
\hline $\mathrm{C}$ & 5.260193 & 2.282529 & 0.414442 \\
\hline $\mathrm{H}$ & 4.785953 & 4.099884 & 1.613465 \\
\hline $\mathrm{H}$ & 6.311099 & 2.297202 & 0.177952 \\
\hline $\mathrm{N}$ & -3.176258 & -1.473766 & -0.416487 \\
\hline $\mathrm{C}$ & -3.202357 & -2.616748 & -1.094880 \\
\hline $\mathrm{C}$ & -4.484256 & -3.184737 & -1.135835 \\
\hline $\mathrm{C}$ & -5.260208 & -2.282546 & -0.414447 \\
\hline $\mathrm{H}$ & -4.785968 & -4.099901 & -1.613470 \\
\hline $\mathrm{H}$ & -6.311115 & -2.297219 & -0.177957 \\
\hline $\mathrm{N}$ & -3.176258 & 1.473766 & -0.416487 \\
\hline $\mathrm{C}$ & -3.202357 & 2.616748 & -1.094880 \\
\hline $\mathrm{C}$ & -4.484256 & 3.184737 & -1.135835 \\
\hline $\mathrm{C}$ & -5.260208 & 2.282546 & -0.414447 \\
\hline $\mathrm{H}$ & -4.785968 & 4.099901 & -1.613470 \\
\hline $\mathrm{H}$ & -6.311115 & 2.297219 & -0.177957 \\
\hline $\mathrm{F}$ & 0.918965 & -0.000017 & -3.723751 \\
\hline $\mathrm{F}$ & -0.918980 & 0.000000 & 3.723747 \\
\hline $\mathrm{F}$ & 2.057023 & -3.109004 & 1.656969 \\
\hline $\mathrm{F}$ & 2.057023 & 3.108971 & 1.656969 \\
\hline $\mathrm{F}$ & -2.057038 & -3.108988 & -1.656974 \\
\hline $\mathrm{F}$ & -2.057038 & 3.108988 & -1.656974 \\
\hline
\end{tabular}




$$
\mathbf{1 a}-\left[\mathrm{Cu}_{2}(\square-\mathrm{O})_{2}\left(\mathrm{Tp}^{3 C l}\right)_{2}\right]
$$

\begin{tabular}{|c|c|c|c|}
\hline $\mathrm{Cu}$ & 1.828418 & -0.000163 & 0.000185 \\
\hline $\mathrm{Cu}$ & -1.828895 & -0.000163 & 0.000185 \\
\hline 0 & -0.000239 & 0.699500 & 0.000185 \\
\hline 0 & -0.000239 & -0.699826 & 0.000185 \\
\hline B & 4.825520 & 0.000000 & -0.803587 \\
\hline B & -4.825520 & 0.000000 & 0.803587 \\
\hline $\mathrm{H}$ & 5.996294 & 0.000000 & -1.059699 \\
\hline $\mathrm{H}$ & -5.996294 & 0.000000 & 1.059699 \\
\hline $\mathrm{N}$ & 4.028834 & 0.000000 & -2.129315 \\
\hline $\mathrm{N}$ & 4.459162 & -1.262380 & 0.028076 \\
\hline $\mathrm{N}$ & 4.459162 & 1.262380 & 0.028076 \\
\hline $\mathrm{N}$ & -4.028834 & 0.000000 & 2.129315 \\
\hline $\mathrm{N}$ & -4.459162 & -1.262380 & -0.028076 \\
\hline $\mathrm{N}$ & -4.459162 & 1.262380 & -0.028076 \\
\hline $\mathrm{N}$ & 2.659786 & 0.000000 & -2.128863 \\
\hline $\mathrm{C}$ & 2.306635 & 0.000000 & -3.410211 \\
\hline $\mathrm{C}$ & 3.416952 & 0.000000 & -4.275889 \\
\hline $\mathrm{C}$ & 4.503967 & 0.000000 & -3.413527 \\
\hline $\mathrm{H}$ & 3.413279 & 0.000000 & -5.351810 \\
\hline $\mathrm{H}$ & 5.562185 & 0.000000 & -3.616640 \\
\hline $\mathrm{N}$ & -2.659786 & 0.000000 & 2.129782 \\
\hline $\mathrm{C}$ & -2.307495 & 0.000000 & 3.411366 \\
\hline $\mathrm{C}$ & -3.418393 & 0.000000 & 4.276299 \\
\hline $\mathrm{C}$ & -4.504829 & 0.000000 & 3.413207 \\
\hline $\mathrm{H}$ & -3.415442 & 0.000000 & 5.352222 \\
\hline $\mathrm{H}$ & -5.563183 & 0.000000 & 3.615610 \\
\hline $\mathrm{N}$ & 3.174218 & -1.484841 & 0.441599 \\
\hline $\mathrm{C}$ & 3.196779 & -2.633629 & 1.120560 \\
\hline $\mathrm{C}$ & 4.487315 & -3.185191 & 1.166606 \\
\hline $\mathrm{C}$ & 5.264359 & -2.277673 & 0.456360 \\
\hline $\mathrm{H}$ & 4.793005 & -4.099491 & 1.644036 \\
\hline $\mathrm{H}$ & 6.317421 & -2.283062 & 0.229207 \\
\hline $\mathrm{N}$ & 3.174218 & 1.484841 & 0.441599 \\
\hline $\mathrm{C}$ & 3.196779 & 2.633629 & 1.120560 \\
\hline $\mathrm{C}$ & 4.487315 & 3.185191 & 1.166606 \\
\hline $\mathrm{C}$ & 5.264359 & 2.277673 & 0.456360 \\
\hline $\mathrm{H}$ & 4.793005 & 4.099491 & 1.644036 \\
\hline $\mathrm{H}$ & 6.317421 & 2.283062 & 0.229207 \\
\hline $\mathrm{N}$ & -3.174218 & -1.484841 & -0.441599 \\
\hline $\mathrm{C}$ & -3.196779 & -2.633629 & -1.120560 \\
\hline $\mathrm{C}$ & -4.487315 & -3.185191 & -1.166606 \\
\hline $\mathrm{C}$ & -5.264359 & -2.277673 & -0.456360 \\
\hline $\mathrm{H}$ & -4.793005 & -4.099491 & -1.644036 \\
\hline $\mathrm{H}$ & -6.317421 & -2.283062 & -0.229207 \\
\hline $\mathrm{N}$ & -3.174218 & 1.484841 & -0.441599 \\
\hline $\mathrm{C}$ & -3.196779 & 2.633629 & -1.120560 \\
\hline $\mathrm{C}$ & -4.487315 & 3.185191 & -1.166606 \\
\hline $\mathrm{C}$ & -5.264359 & 2.277673 & -0.456360 \\
\hline $\mathrm{H}$ & -4.793005 & 4.099491 & -1.644036 \\
\hline $\mathrm{H}$ & -6.317421 & 2.283062 & -0.229207 \\
\hline $\mathrm{Cl}$ & 0.584415 & 0.000000 & -3.894089 \\
\hline $\mathrm{Cl}$ & -0.585601 & 0.000000 & 3.896401 \\
\hline $\mathrm{Cl}$ & 1.716532 & -3.309534 & 1.846750 \\
\hline $\mathrm{Cl}$ & 1.716532 & 3.309534 & 1.846750 \\
\hline $\mathrm{Cl}$ & -1.716532 & -3.309534 & -1.846750 \\
\hline $\mathrm{Cl}$ & -1.716532 & 3.309534 & -1.846750 \\
\hline
\end{tabular}




$$
\mathbf{1 a}-\left[\mathrm{Cu}_{2}(\square-\mathrm{O})_{2}\left(\mathrm{Tp}^{3 B r}\right)_{2}\right]
$$

\begin{tabular}{|c|c|c|c|}
\hline $\mathrm{Cu}$ & 1.833505 & 0.000000 & 0.000000 \\
\hline $\mathrm{Cu}$ & -1.833505 & 0.000000 & 0.000000 \\
\hline 0 & 0.000000 & 0.698989 & 0.000000 \\
\hline 0 & 0.000000 & -0.698989 & 0.000000 \\
\hline B & 4.848358 & 0.000000 & -0.714491 \\
\hline B & -4.848358 & 0.000000 & 0.714491 \\
\hline $\mathrm{H}$ & 6.027033 & 0.000000 & -0.932554 \\
\hline $\mathrm{H}$ & -6.027033 & 0.000000 & 0.932554 \\
\hline $\mathrm{N}$ & 4.094378 & 0.000000 & -2.063901 \\
\hline $\mathrm{N}$ & 4.459836 & -1.263497 & 0.103575 \\
\hline $\mathrm{N}$ & 4.459836 & 1.263497 & 0.103575 \\
\hline $\mathrm{N}$ & -4.094378 & 0.000000 & 2.063901 \\
\hline $\mathrm{N}$ & -4.459836 & -1.263497 & -0.103575 \\
\hline $\mathrm{N}$ & -4.459836 & 1.263497 & -0.103575 \\
\hline $\mathrm{N}$ & 2.725500 & 0.000000 & -2.112569 \\
\hline $\mathrm{C}$ & 2.416591 & 0.000000 & -3.408269 \\
\hline $\mathrm{C}$ & 3.561595 & 0.000000 & -4.230892 \\
\hline $\mathrm{C}$ & 4.616090 & 0.000000 & -3.329556 \\
\hline $\mathrm{H}$ & 3.599787 & 0.000000 & -5.306246 \\
\hline $\mathrm{H}$ & 5.681179 & 0.000000 & -3.493288 \\
\hline $\mathrm{N}$ & -2.725500 & 0.000000 & 2.112569 \\
\hline $\mathrm{C}$ & -2.416591 & 0.000000 & 3.408269 \\
\hline $\mathrm{C}$ & -3.561595 & 0.000000 & 4.230892 \\
\hline $\mathrm{C}$ & -4.616090 & 0.000000 & 3.329556 \\
\hline $\mathrm{H}$ & -3.599787 & 0.000000 & 5.306246 \\
\hline $\mathrm{H}$ & -5.681179 & 0.000000 & 3.493288 \\
\hline $\mathrm{N}$ & 3.163916 & -1.493441 & 0.479128 \\
\hline $\mathrm{C}$ & 3.170481 & -2.645842 & 1.158490 \\
\hline $\mathrm{C}$ & 4.466036 & -3.187508 & 1.238696 \\
\hline $\mathrm{C}$ & 5.258091 & -2.274650 & 0.553052 \\
\hline $\mathrm{H}$ & 4.765813 & -4.100607 & 1.722487 \\
\hline $\mathrm{H}$ & 6.317332 & -2.273185 & 0.356220 \\
\hline $\mathrm{N}$ & 3.163916 & 1.493441 & 0.479128 \\
\hline $\mathrm{C}$ & 3.170481 & 2.645842 & 1.158490 \\
\hline $\mathrm{C}$ & 4.466036 & 3.187508 & 1.238696 \\
\hline $\mathrm{C}$ & 5.258091 & 2.274650 & 0.553052 \\
\hline $\mathrm{H}$ & 4.765813 & 4.100607 & 1.722487 \\
\hline $\mathrm{H}$ & 6.317332 & 2.273185 & 0.356220 \\
\hline $\mathrm{N}$ & -3.163916 & -1.493441 & -0.479128 \\
\hline $\mathrm{C}$ & -3.170481 & -2.645842 & -1.158490 \\
\hline $\mathrm{C}$ & -4.466036 & -3.187508 & -1.238696 \\
\hline $\mathrm{C}$ & -5.258091 & -2.274650 & -0.553052 \\
\hline $\mathrm{H}$ & -4.765813 & -4.100607 & -1.722487 \\
\hline $\mathrm{H}$ & -6.317332 & -2.273185 & -0.356220 \\
\hline $\mathrm{N}$ & -3.163916 & 1.493441 & -0.479128 \\
\hline $\mathrm{C}$ & -3.170481 & 2.645842 & -1.158490 \\
\hline $\mathrm{C}$ & -4.466036 & 3.187508 & -1.238696 \\
\hline $\mathrm{C}$ & -5.258091 & 2.274650 & -0.553052 \\
\hline $\mathrm{H}$ & -4.765813 & 4.100607 & -1.722487 \\
\hline $\mathrm{H}$ & -6.317332 & 2.273185 & -0.356220 \\
\hline $\mathrm{Br}$ & 0.567832 & 0.000000 & -4.013002 \\
\hline $\mathrm{Br}$ & -0.567832 & 0.000000 & 4.013002 \\
\hline $\mathrm{Br}$ & 1.550282 & -3.403381 & 1.905619 \\
\hline $\mathrm{Br}$ & 1.550282 & 3.403381 & 1.905619 \\
\hline $\mathrm{Br}$ & -1.550282 & -3.403381 & -1.905619 \\
\hline $\mathrm{Br}$ & -1.550282 & 3.403381 & -1.905619 \\
\hline
\end{tabular}




$$
\mathbf{1 a}-\left[\mathrm{Cu}_{2}(\square-\mathrm{O})_{2}\left(\mathrm{Tp}^{3 I}\right)_{2}\right]
$$

\begin{tabular}{|c|c|c|c|}
\hline $\mathrm{Cu}$ & 1.841633 & 0.000000 & 0.000000 \\
\hline $\mathrm{Cu}$ & -1.841633 & 0.000000 & 0.000000 \\
\hline 0 & 0.000000 & 0.698251 & 0.000000 \\
\hline 0 & 0.000000 & -0.698251 & 0.000000 \\
\hline B & 4.860384 & 0.000000 & -0.647155 \\
\hline B & -4.860384 & 0.000000 & 0.647155 \\
\hline $\mathrm{H}$ & 6.044604 & 0.000000 & -0.834706 \\
\hline $\mathrm{H}$ & -6.044604 & 0.000000 & 0.834706 \\
\hline $\mathrm{N}$ & 4.146193 & 0.000000 & -2.016918 \\
\hline $\mathrm{N}$ & 4.457589 & -1.264404 & 0.158356 \\
\hline $\mathrm{N}$ & 4.457589 & 1.264404 & 0.158356 \\
\hline $\mathrm{N}$ & -4.146193 & 0.000000 & 2.016918 \\
\hline $\mathrm{N}$ & -4.457589 & -1.264404 & -0.158356 \\
\hline $\mathrm{N}$ & -4.457589 & 1.264404 & -0.158356 \\
\hline $\mathrm{N}$ & 2.779363 & 0.000000 & -2.117848 \\
\hline $\mathrm{C}$ & 2.517000 & 0.000000 & -3.428871 \\
\hline $\mathrm{C}$ & 3.699880 & 0.000000 & -4.200532 \\
\hline $\mathrm{C}$ & 4.717714 & 0.000000 & -3.259981 \\
\hline $\mathrm{H}$ & 3.783274 & 0.000000 & -5.273573 \\
\hline $\mathrm{H}$ & 5.788552 & 0.000000 & -3.380519 \\
\hline $\mathrm{N}$ & -2.779363 & 0.000000 & 2.117848 \\
\hline $\mathrm{C}$ & -2.517000 & 0.000000 & 3.428871 \\
\hline $\mathrm{C}$ & -3.699880 & 0.000000 & 4.200532 \\
\hline $\mathrm{C}$ & -4.717714 & 0.000000 & 3.259981 \\
\hline $\mathrm{H}$ & -3.783274 & 0.000000 & 5.273573 \\
\hline $\mathrm{H}$ & -5.788552 & 0.000000 & 3.380519 \\
\hline $\mathrm{N}$ & 3.156496 & -1.508090 & 0.507366 \\
\hline $\mathrm{C}$ & 3.156937 & -2.666389 & 1.186771 \\
\hline $\mathrm{C}$ & 4.461949 & -3.188799 & 1.286543 \\
\hline $\mathrm{C}$ & 5.257887 & -2.267250 & 0.619892 \\
\hline $\mathrm{H}$ & 4.765369 & -4.099693 & 1.772831 \\
\hline $\mathrm{H}$ & 6.320605 & -2.253095 & 0.443280 \\
\hline $\mathrm{N}$ & 3.156496 & 1.508090 & 0.507366 \\
\hline $\mathrm{C}$ & 3.156937 & 2.666389 & 1.186771 \\
\hline $\mathrm{C}$ & 4.461949 & 3.188799 & 1.286543 \\
\hline $\mathrm{C}$ & 5.257887 & 2.267250 & 0.619892 \\
\hline $\mathrm{H}$ & 4.765369 & 4.099693 & 1.772831 \\
\hline $\mathrm{H}$ & 6.320605 & 2.253095 & 0.443280 \\
\hline $\mathrm{N}$ & -3.156496 & -1.508090 & -0.507366 \\
\hline $\mathrm{C}$ & -3.156937 & -2.666389 & -1.186771 \\
\hline $\mathrm{C}$ & -4.461949 & -3.188799 & -1.286543 \\
\hline $\mathrm{C}$ & -5.257887 & -2.267250 & -0.619892 \\
\hline $\mathrm{H}$ & -4.765369 & -4.099693 & -1.772831 \\
\hline $\mathrm{H}$ & -6.320605 & -2.253095 & -0.443280 \\
\hline $\mathrm{N}$ & -3.156496 & 1.508090 & -0.507366 \\
\hline $\mathrm{C}$ & -3.156937 & 2.666389 & -1.186771 \\
\hline $\mathrm{C}$ & -4.461949 & 3.188799 & -1.286543 \\
\hline $\mathrm{C}$ & -5.257887 & 2.267250 & -0.619892 \\
\hline $\mathrm{H}$ & -4.765369 & 4.099693 & -1.772831 \\
\hline $\mathrm{H}$ & -6.320605 & 2.253095 & -0.443280 \\
\hline$I$ & 0.549442 & 0.000000 & -4.195418 \\
\hline I & -0.549442 & 0.000000 & 4.195418 \\
\hline$I$ & 1.409683 & -3.538302 & 1.974969 \\
\hline $\mathrm{I}$ & 1.409683 & 3.538302 & 1.974969 \\
\hline$I$ & -1.409683 & -3.538302 & -1.974969 \\
\hline$I$ & -1.409683 & 3.538302 & -1.974969 \\
\hline
\end{tabular}




$$
\mathbf{1 a}-\left[\mathrm{Cu}_{2}(\square-\mathrm{O})_{2}\left(\mathrm{Tp}^{5 F}\right)_{2}\right]
$$

\begin{tabular}{|c|c|c|c|}
\hline $\mathrm{Cu}$ & 1.828192 & 0.000000 & 0.000000 \\
\hline $\mathrm{Cu}$ & -1.828192 & 0.000000 & 0.000000 \\
\hline 0 & 0.000000 & 0.705724 & 0.000000 \\
\hline 0 & 0.000000 & -0.705724 & 0.000000 \\
\hline B & 4.840906 & 0.000000 & -0.684070 \\
\hline B & -4.840906 & 0.000000 & 0.684070 \\
\hline $\mathrm{H}$ & 6.010253 & 0.000000 & -0.892011 \\
\hline $\mathrm{H}$ & -6.010253 & 0.000000 & 0.892011 \\
\hline $\mathrm{N}$ & 4.070785 & 0.000000 & -2.027616 \\
\hline $\mathrm{N}$ & 4.424060 & -1.258961 & 0.135763 \\
\hline $\mathrm{N}$ & 4.424060 & 1.258961 & 0.135763 \\
\hline $\mathrm{N}$ & -4.070785 & 0.000000 & 2.027616 \\
\hline $\mathrm{N}$ & -4.424060 & -1.258961 & -0.135763 \\
\hline $\mathrm{N}$ & -4.424060 & 1.258961 & -0.135763 \\
\hline $\mathrm{N}$ & 2.696928 & 0.000000 & -2.082703 \\
\hline $\mathrm{C}$ & 2.362773 & 0.000000 & -3.379443 \\
\hline $\mathrm{C}$ & 3.509122 & 0.000000 & -4.206403 \\
\hline $\mathrm{C}$ & 4.551352 & 0.000000 & -3.300415 \\
\hline $\mathrm{H}$ & 3.571401 & 0.000000 & -5.280276 \\
\hline $\mathrm{F}$ & 5.898342 & 0.000000 & -3.536469 \\
\hline $\mathrm{N}$ & -2.696928 & 0.000000 & 2.082703 \\
\hline $\mathrm{C}$ & -2.362773 & 0.000000 & 3.379443 \\
\hline $\mathrm{C}$ & -3.509122 & 0.000000 & 4.206403 \\
\hline $\mathrm{C}$ & -4.551352 & 0.000000 & 3.300415 \\
\hline $\mathrm{H}$ & -3.571401 & 0.000000 & 5.280276 \\
\hline $\mathrm{F}$ & -5.898342 & 0.000000 & 3.536469 \\
\hline $\mathrm{N}$ & 3.121234 & -1.484007 & 0.500739 \\
\hline $\mathrm{C}$ & 3.085209 & -2.635480 & 1.189186 \\
\hline $\mathrm{C}$ & 4.376024 & -3.192740 & 1.289721 \\
\hline $\mathrm{C}$ & 5.172140 & -2.286318 & 0.611258 \\
\hline $\mathrm{H}$ & 4.682599 & -4.102724 & 1.774205 \\
\hline $\mathrm{F}$ & 6.518249 & -2.322492 & 0.393311 \\
\hline $\mathrm{N}$ & 3.121234 & 1.484007 & 0.500739 \\
\hline $\mathrm{C}$ & 3.085209 & 2.635480 & 1.189186 \\
\hline $\mathrm{C}$ & 4.376024 & 3.192740 & 1.289721 \\
\hline $\mathrm{C}$ & 5.172140 & 2.286318 & 0.611258 \\
\hline $\mathrm{H}$ & 4.682599 & 4.102724 & 1.774205 \\
\hline $\mathrm{F}$ & 6.518249 & 2.322492 & 0.393311 \\
\hline $\mathrm{N}$ & -3.121234 & -1.484007 & -0.500739 \\
\hline $\mathrm{C}$ & -3.085209 & -2.635480 & -1.189186 \\
\hline $\mathrm{C}$ & -4.376024 & -3.192740 & -1.289721 \\
\hline $\mathrm{C}$ & -5.172140 & -2.286318 & -0.611258 \\
\hline $\mathrm{H}$ & -4.682599 & -4.102724 & -1.774205 \\
\hline $\mathrm{F}$ & -6.518249 & -2.322492 & -0.393311 \\
\hline $\mathrm{N}$ & -3.121234 & 1.484007 & -0.500739 \\
\hline $\mathrm{C}$ & -3.085209 & 2.635480 & -1.189186 \\
\hline $\mathrm{C}$ & -4.376024 & 3.192740 & -1.289721 \\
\hline $\mathrm{C}$ & -5.172140 & 2.286318 & -0.611258 \\
\hline $\mathrm{H}$ & -4.682599 & 4.102724 & -1.774205 \\
\hline $\mathrm{F}$ & -6.518249 & 2.322492 & -0.393311 \\
\hline $\mathrm{H}$ & 1.324327 & 0.000000 & -3.673763 \\
\hline $\mathrm{H}$ & -1.324327 & 0.000000 & 3.673763 \\
\hline $\mathrm{H}$ & 2.152503 & -3.012722 & 1.578493 \\
\hline $\mathrm{H}$ & 2.152503 & 3.012722 & 1.578493 \\
\hline $\mathrm{H}$ & -2.152503 & -3.012722 & -1.578493 \\
\hline $\mathrm{H}$ & -2.152503 & 3.012722 & -1.578493 \\
\hline
\end{tabular}




$$
\mathbf{1 a}-\left[\mathrm{Cu}_{2}(\square-\mathrm{O})_{2}\left(\mathrm{Tp}^{3 F, 5 F}\right)_{2}\right]
$$

\begin{tabular}{|c|c|c|c|}
\hline $\mathrm{Cu}$ & 1.823205 & 0.000000 & -0.000013 \\
\hline $\mathrm{Cu}$ & -1.823195 & 0.000000 & -0.000013 \\
\hline $\mathrm{O}$ & 0.000005 & 0.701050 & -0.000013 \\
\hline $\mathrm{O}$ & 0.000005 & -0.701050 & -0.000013 \\
\hline B & 4.836855 & 0.000000 & -0.875191 \\
\hline B & -4.836860 & 0.000000 & 0.875204 \\
\hline $\mathrm{H}$ & 5.991290 & 0.000000 & -1.152094 \\
\hline $\mathrm{H}$ & -5.991295 & 0.000000 & 1.152107 \\
\hline $\mathrm{N}$ & 3.991732 & 0.000000 & -2.171392 \\
\hline $\mathrm{N}$ & 4.467617 & -1.257689 & -0.032100 \\
\hline $\mathrm{N}$ & 4.467617 & 1.257689 & -0.032100 \\
\hline $\mathrm{N}$ & -3.991737 & 0.000000 & 2.171405 \\
\hline $\mathrm{N}$ & -4.467621 & -1.257689 & 0.032113 \\
\hline $\mathrm{N}$ & -4.467621 & 1.257689 & 0.032113 \\
\hline $\mathrm{N}$ & 2.611086 & 0.000000 & -2.131837 \\
\hline $\mathrm{C}$ & 2.223308 & 0.000000 & -3.396332 \\
\hline $\mathrm{C}$ & 3.290026 & 0.000000 & -4.315512 \\
\hline $\mathrm{C}$ & 4.386175 & 0.000000 & -3.473517 \\
\hline $\mathrm{H}$ & 3.262662 & 0.000000 & -5.389187 \\
\hline $\mathrm{F}$ & 5.711474 & 0.000000 & -3.797039 \\
\hline $\mathrm{N}$ & -2.611090 & 0.000000 & 2.131850 \\
\hline $\mathrm{C}$ & -2.223313 & 0.000000 & 3.396346 \\
\hline $\mathrm{C}$ & -3.290031 & 0.000000 & 4.315525 \\
\hline $\mathrm{C}$ & -4.386180 & 0.000000 & 3.473530 \\
\hline $\mathrm{H}$ & -3.262667 & 0.000000 & 5.389201 \\
\hline $\mathrm{F}$ & -5.711479 & 0.000000 & 3.797052 \\
\hline $\mathrm{N}$ & 3.177894 & -1.474546 & 0.407353 \\
\hline $\mathrm{C}$ & 3.206711 & -2.615932 & 1.085193 \\
\hline $\mathrm{C}$ & 4.483881 & -3.196724 & 1.127285 \\
\hline $\mathrm{C}$ & 5.236370 & -2.288118 & 0.401692 \\
\hline $\mathrm{H}$ & 4.798138 & -4.108925 & 1.599164 \\
\hline $\mathrm{F}$ & 6.564608 & -2.329666 & 0.108027 \\
\hline $\mathrm{N}$ & 3.177894 & 1.474546 & 0.407353 \\
\hline C & 3.206711 & 2.615932 & 1.085193 \\
\hline $\mathrm{C}$ & 4.483881 & 3.196724 & 1.127285 \\
\hline $\mathrm{C}$ & 5.236370 & 2.288118 & 0.401692 \\
\hline $\mathrm{H}$ & 4.798138 & 4.108925 & 1.599164 \\
\hline $\mathrm{F}$ & 6.564608 & 2.329666 & 0.108027 \\
\hline $\mathrm{N}$ & -3.177899 & -1.474546 & -0.407340 \\
\hline $\mathrm{C}$ & -3.206716 & -2.615932 & -1.085179 \\
\hline $\mathrm{C}$ & -4.483886 & -3.196724 & -1.127272 \\
\hline $\mathrm{C}$ & -5.236375 & -2.288118 & -0.401679 \\
\hline $\mathrm{H}$ & -4.798143 & -4.108925 & -1.599151 \\
\hline $\mathrm{F}$ & -6.564612 & -2.329666 & -0.108014 \\
\hline $\mathrm{N}$ & -3.177899 & 1.474546 & -0.407340 \\
\hline $\mathrm{C}$ & -3.206716 & 2.615932 & -1.085179 \\
\hline $\mathrm{C}$ & -4.483886 & 3.196724 & -1.127272 \\
\hline $\mathrm{C}$ & -5.236375 & 2.288118 & -0.401679 \\
\hline $\mathrm{H}$ & -4.798143 & 4.108925 & -1.599151 \\
\hline $\mathrm{F}$ & -6.564612 & 2.329666 & -0.108014 \\
\hline $\mathrm{F}$ & 0.889251 & 0.000000 & -3.703158 \\
\hline $\mathrm{F}$ & -0.889256 & 0.000000 & 3.703171 \\
\hline $\mathrm{F}$ & 2.064779 & -3.103051 & 1.651334 \\
\hline $\mathrm{F}$ & 2.064779 & 3.103051 & 1.651334 \\
\hline $\mathrm{F}$ & -2.064784 & -3.103051 & -1.651321 \\
\hline $\mathrm{F}$ & -2.064784 & 3.103051 & -1.651321 \\
\hline
\end{tabular}




$$
\mathbf{1 a}-\left[\mathrm{Cu}_{2}(\square-\mathrm{O})_{2}\left(\mathrm{Tp}^{3 \mathrm{CH}_{3}}\right)_{2}\right]
$$

\begin{tabular}{|c|c|c|c|}
\hline $\mathrm{Cu}$ & 1.844874 & 0.000000 & 0.000000 \\
\hline $\mathrm{Cu}$ & -1.844874 & 0.000000 & 0.000000 \\
\hline 0 & 0.000000 & 0.704608 & 0.000000 \\
\hline 0 & 0.000000 & -0.704608 & 0.000000 \\
\hline B & 4.817794 & 0.000000 & -0.701723 \\
\hline B & -4.817794 & 0.000000 & 0.701723 \\
\hline $\mathrm{H}$ & 5.995441 & 0.000000 & -0.930698 \\
\hline $\mathrm{H}$ & -5.995441 & 0.000000 & 0.930698 \\
\hline $\mathrm{N}$ & 4.049541 & 0.000000 & -2.044861 \\
\hline $\mathrm{N}$ & 4.429962 & -1.261629 & 0.123699 \\
\hline $\mathrm{N}$ & 4.429962 & 1.261629 & 0.123699 \\
\hline $\mathrm{N}$ & -4.049541 & 0.000000 & 2.044861 \\
\hline $\mathrm{N}$ & -4.429962 & -1.261629 & -0.123699 \\
\hline $\mathrm{N}$ & -4.429962 & 1.261629 & -0.123699 \\
\hline $\mathrm{N}$ & 2.681912 & 0.000000 & -2.084734 \\
\hline C & 2.322300 & 0.000000 & -3.378741 \\
\hline $\mathrm{C}$ & 3.480715 & 0.000000 & -4.198001 \\
\hline C & 4.551370 & 0.000000 & -3.316961 \\
\hline $\mathrm{H}$ & 3.519819 & 0.000000 & -5.275639 \\
\hline $\mathrm{H}$ & 5.614403 & 0.000000 & -3.495337 \\
\hline $\mathrm{N}$ & -2.681912 & 0.000000 & 2.084734 \\
\hline $\mathrm{C}$ & -2.322300 & 0.000000 & 3.378741 \\
\hline $\mathrm{C}$ & -3.480715 & 0.000000 & 4.198001 \\
\hline $\mathrm{C}$ & -4.551370 & 0.000000 & 3.316961 \\
\hline $\mathrm{H}$ & -3.519819 & 0.000000 & 5.275639 \\
\hline $\mathrm{H}$ & -5.614403 & 0.000000 & 3.495337 \\
\hline $\mathrm{N}$ & 3.137509 & -1.484895 & 0.507724 \\
\hline $\mathrm{C}$ & 3.101804 & -2.639365 & 1.200977 \\
\hline C & 4.409964 & -3.176107 & 1.265707 \\
\hline C & 5.215745 & -2.279418 & 0.575971 \\
\hline $\mathrm{H}$ & 4.714147 & -4.089972 & 1.749839 \\
\hline $\mathrm{H}$ & 6.274660 & -2.290207 & 0.376495 \\
\hline $\mathrm{N}$ & 3.137509 & 1.484895 & 0.507724 \\
\hline C & 3.101804 & 2.639365 & 1.200977 \\
\hline $\mathrm{C}$ & 4.409964 & 3.176107 & 1.265707 \\
\hline C & 5.215745 & 2.279418 & 0.575971 \\
\hline $\mathrm{H}$ & 4.714147 & 4.089972 & 1.749839 \\
\hline $\mathrm{H}$ & 6.274660 & 2.290207 & 0.376495 \\
\hline $\mathrm{N}$ & -3.137509 & -1.484895 & -0.507724 \\
\hline $\mathrm{C}$ & -3.101804 & -2.639365 & -1.200977 \\
\hline $\mathrm{C}$ & -4.409964 & -3.176107 & -1.265707 \\
\hline $\mathrm{C}$ & -5.215745 & -2.279418 & -0.575971 \\
\hline $\mathrm{H}$ & -4.714147 & -4.089972 & -1.749839 \\
\hline $\mathrm{H}$ & -6.274660 & -2.290207 & -0.376495 \\
\hline $\mathrm{N}$ & -3.137509 & 1.484895 & -0.507724 \\
\hline $\mathrm{C}$ & -3.101804 & 2.639365 & -1.200977 \\
\hline $\mathrm{C}$ & -4.409964 & 3.176107 & -1.265707 \\
\hline $\mathrm{C}$ & -5.215745 & 2.279418 & -0.575971 \\
\hline $\mathrm{H}$ & -4.714147 & 4.089972 & -1.749839 \\
\hline $\mathrm{H}$ & -6.274660 & 2.290207 & -0.376495 \\
\hline C & 0.879753 & 0.000000 & -3.785934 \\
\hline $\mathrm{C}$ & -0.879753 & 0.000000 & 3.785934 \\
\hline $\mathrm{C}$ & 1.826413 & -3.180477 & 1.770637 \\
\hline $\mathrm{C}$ & 1.826413 & 3.180477 & 1.770637 \\
\hline $\mathrm{C}$ & -1.826413 & -3.180477 & -1.770637 \\
\hline $\mathrm{C}$ & -1.826413 & 3.180477 & -1.770637 \\
\hline $\mathrm{H}$ & 0.223199 & 0.000000 & -2.908700 \\
\hline $\mathrm{H}$ & 0.639896 & 0.885600 & -4.384920 \\
\hline $\mathrm{H}$ & 0.639896 & -0.885600 & -4.384920 \\
\hline $\mathrm{H}$ & -0.223199 & 0.000000 & 2.908700 \\
\hline $\mathrm{H}$ & -0.639896 & 0.885600 & 4.384920 \\
\hline $\mathrm{H}$ & -0.639896 & -0.885600 & 4.384920 \\
\hline $\mathrm{H}$ & 0.994449 & -2.488755 & 1.599395 \\
\hline $\mathrm{H}$ & 1.565802 & -4.139732 & 1.310338 \\
\hline
\end{tabular}




$\begin{array}{lrrr}\mathrm{H} & 1.914281 & -3.339210 & 2.850938 \\ \mathrm{H} & 0.994449 & 2.488755 & 1.599395 \\ \mathrm{H} & 1.914281 & 3.339210 & 2.850938 \\ \mathrm{H} & 1.565802 & 4.139732 & 1.310338 \\ \mathrm{H} & -0.994449 & -2.488755 & -1.599395 \\ \mathrm{H} & -1.565802 & -4.139732 & -1.310338 \\ \mathrm{H} & -1.914281 & -3.339210 & -2.850938 \\ \mathrm{H} & -0.994449 & 2.488755 & -1.599395 \\ \mathrm{H} & -1.914281 & 3.339210 & -2.850938 \\ \mathrm{H} & -1.565802 & 4.139732 & -1.310338\end{array}$




$$
\mathbf{1 a}-\left[\mathrm{Cu}_{2}(\square-\mathrm{O})_{2}\left(\mathrm{Tp}^{3 C F_{3}}\right)_{2}\right]
$$

\begin{tabular}{|c|c|c|c|}
\hline $\mathrm{Cu}$ & 1.816746 & 0.000000 & 0.000000 \\
\hline $\mathrm{Cu}$ & -1.816746 & 0.000000 & 0.000000 \\
\hline $\mathrm{O}$ & 0.000000 & 0.714246 & 0.000000 \\
\hline 0 & 0.000000 & -0.714246 & 0.000000 \\
\hline B & 4.803182 & 0.000000 & -0.919348 \\
\hline B & -4.803182 & 0.000000 & 0.919348 \\
\hline $\mathrm{H}$ & 5.960262 & 0.000000 & -1.223663 \\
\hline $\mathrm{H}$ & -5.960262 & 0.000000 & 1.223663 \\
\hline $\mathrm{N}$ & 3.967915 & 0.000000 & -2.219306 \\
\hline $\mathrm{N}$ & 4.463816 & -1.257202 & -0.068949 \\
\hline $\mathrm{N}$ & 4.463816 & 1.257202 & -0.068949 \\
\hline $\mathrm{N}$ & -3.967915 & 0.000000 & 2.219306 \\
\hline $\mathrm{N}$ & -4.463816 & -1.257202 & 0.068949 \\
\hline $\mathrm{N}$ & -4.463816 & 1.257202 & 0.068949 \\
\hline $\mathrm{N}$ & 2.610860 & 0.000000 & -2.196805 \\
\hline $\mathrm{C}$ & 2.223716 & 0.000000 & -3.480601 \\
\hline $\mathrm{C}$ & 3.335058 & 0.000000 & -4.352435 \\
\hline $\mathrm{C}$ & 4.432072 & 0.000000 & -3.510333 \\
\hline $\mathrm{H}$ & 3.324379 & 0.000000 & -5.429340 \\
\hline $\mathrm{H}$ & 5.487617 & 0.000000 & -3.724404 \\
\hline $\mathrm{N}$ & -2.610860 & 0.000000 & 2.196805 \\
\hline $\mathrm{C}$ & -2.223716 & 0.000000 & 3.480601 \\
\hline $\mathrm{C}$ & -3.335058 & 0.000000 & 4.352435 \\
\hline $\mathrm{C}$ & -4.432072 & 0.000000 & 3.510333 \\
\hline $\mathrm{H}$ & -3.324379 & 0.000000 & 5.429340 \\
\hline $\mathrm{H}$ & -5.487617 & 0.000000 & 3.724404 \\
\hline $\mathrm{N}$ & 3.207069 & -1.478591 & 0.395613 \\
\hline $\mathrm{C}$ & 3.253328 & -2.633561 & 1.082697 \\
\hline $\mathrm{C}$ & 4.552722 & -3.171811 & 1.066824 \\
\hline $\mathrm{C}$ & 5.295715 & -2.266224 & 0.323148 \\
\hline $\mathrm{H}$ & 4.883702 & -4.083945 & 1.532006 \\
\hline $\mathrm{H}$ & 6.336852 & -2.264757 & 0.048438 \\
\hline $\mathrm{N}$ & 3.207069 & 1.478591 & 0.395613 \\
\hline $\mathrm{C}$ & 3.253328 & 2.633561 & 1.082697 \\
\hline C & 4.552722 & 3.171811 & 1.066824 \\
\hline $\mathrm{C}$ & 5.295715 & 2.266224 & 0.323148 \\
\hline $\mathrm{H}$ & 4.883702 & 4.083945 & 1.532006 \\
\hline $\mathrm{H}$ & 6.336852 & 2.264757 & 0.048438 \\
\hline $\mathrm{N}$ & -3.207069 & -1.478591 & -0.395613 \\
\hline $\mathrm{C}$ & -3.253328 & -2.633561 & -1.082697 \\
\hline $\mathrm{C}$ & -4.552722 & -3.171811 & -1.066824 \\
\hline $\mathrm{C}$ & -5.295715 & -2.266224 & -0.323148 \\
\hline $\mathrm{H}$ & -4.883702 & -4.083945 & -1.532006 \\
\hline $\mathrm{H}$ & -6.336852 & -2.264757 & -0.048438 \\
\hline $\mathrm{N}$ & -3.207069 & 1.478591 & -0.395613 \\
\hline $\mathrm{C}$ & -3.253328 & 2.633561 & -1.082697 \\
\hline $\mathrm{C}$ & -4.552722 & 3.171811 & -1.066824 \\
\hline $\mathrm{C}$ & -5.295715 & 2.266224 & -0.323148 \\
\hline $\mathrm{H}$ & -4.883702 & 4.083945 & -1.532006 \\
\hline $\mathrm{H}$ & -6.336852 & 2.264757 & -0.048438 \\
\hline $\mathrm{C}$ & 0.801274 & 0.000000 & -3.890978 \\
\hline $\mathrm{C}$ & -0.801274 & 0.000000 & 3.890978 \\
\hline $\mathrm{C}$ & 2.057596 & -3.205202 & 1.743265 \\
\hline $\mathrm{C}$ & 2.057596 & 3.205202 & 1.743265 \\
\hline $\mathrm{C}$ & -2.057596 & -3.205202 & -1.743265 \\
\hline $\mathrm{C}$ & -2.057596 & 3.205202 & -1.743265 \\
\hline $\mathrm{F}$ & -0.057143 & 0.000097 & -2.795564 \\
\hline $\mathrm{F}$ & 0.479437 & 1.117504 & -4.655450 \\
\hline $\mathrm{F}$ & 0.479391 & -1.117601 & -4.655289 \\
\hline $\mathrm{F}$ & 0.057143 & -0.000097 & 2.795564 \\
\hline $\mathrm{F}$ & -0.479391 & 1.117601 & 4.655289 \\
\hline $\mathrm{F}$ & -0.479437 & -1.117504 & 4.655450 \\
\hline $\mathrm{F}$ & 1.550303 & -2.352355 & 2.717068 \\
\hline $\mathrm{F}$ & 1.032712 & -3.444715 & 0.834842 \\
\hline
\end{tabular}




$\begin{array}{lrrr}\mathrm{F} & 2.342740 & -4.414702 & 2.366793 \\ \mathrm{~F} & 1.550303 & 2.352355 & 2.717068 \\ \mathrm{~F} & 2.342740 & 4.414702 & 2.366793 \\ \mathrm{~F} & 1.032712 & 3.444715 & 0.834842 \\ \mathrm{~F} & -1.550303 & -2.352355 & -2.717068 \\ \mathrm{~F} & -1.032712 & -3.444715 & -0.834842 \\ \mathrm{~F} & -2.342740 & -4.414702 & -2.366793 \\ \mathrm{~F} & -1.550303 & 2.352355 & -2.717068 \\ \mathrm{~F} & -2.342740 & 4.414702 & -2.366793 \\ \mathrm{~F} & -1.032712 & 3.444715 & -0.834842\end{array}$


$\mathbf{1 a}-\left[\mathrm{Cu}_{2}(\square-\mathrm{O})_{2}\left(\mathrm{Tp}^{a p-3 C F_{3}}\right)_{2}\right]$

\begin{tabular}{|c|c|c|c|}
\hline $\mathrm{Cu}$ & 1.813374 & 0.000000 & 0.000000 \\
\hline $\mathrm{Cu}$ & -1.813374 & 0.000000 & 0.000000 \\
\hline 0 & 0.000000 & 0.707106 & 0.000000 \\
\hline 0 & 0.000000 & -0.707106 & 0.000000 \\
\hline B & 4.692848 & 0.000000 & -1.113064 \\
\hline B & -4.692848 & 0.000000 & 1.113064 \\
\hline $\mathrm{H}$ & 5.828545 & 0.000000 & -1.494051 \\
\hline $\mathrm{H}$ & -5.828545 & 0.000000 & 1.494051 \\
\hline $\mathrm{N}$ & 3.763706 & 0.000000 & -2.370063 \\
\hline $\mathrm{N}$ & 4.396815 & -1.262688 & -0.258994 \\
\hline $\mathrm{N}$ & 4.396815 & 1.262688 & -0.258994 \\
\hline $\mathrm{N}$ & -3.763706 & 0.000000 & 2.370063 \\
\hline $\mathrm{N}$ & -4.396815 & -1.262688 & 0.258994 \\
\hline $\mathrm{N}$ & -4.396815 & 1.262688 & 0.258994 \\
\hline $\mathrm{N}$ & 2.418331 & 0.000000 & -2.239077 \\
\hline $\mathrm{C}$ & 1.920647 & 0.000000 & -3.483402 \\
\hline $\mathrm{C}$ & 2.952833 & 0.000000 & -4.446722 \\
\hline $\mathrm{C}$ & 4.118246 & 0.000000 & -3.694590 \\
\hline $\mathrm{H}$ & 2.850715 & 0.000000 & -5.518393 \\
\hline $\mathrm{H}$ & 5.152245 & 0.000000 & -3.995918 \\
\hline $\mathrm{N}$ & -2.418331 & 0.000000 & 2.239077 \\
\hline $\mathrm{C}$ & -1.920647 & 0.000000 & 3.483402 \\
\hline $\mathrm{C}$ & -2.952833 & 0.000000 & 4.446722 \\
\hline $\mathrm{C}$ & -4.118246 & 0.000000 & 3.694590 \\
\hline $\mathrm{H}$ & -2.850715 & 0.000000 & 5.518393 \\
\hline $\mathrm{H}$ & -5.152245 & 0.000000 & 3.995918 \\
\hline $\mathrm{N}$ & 3.160767 & -1.473672 & 0.281772 \\
\hline $\mathrm{C}$ & 3.208051 & -2.631577 & 0.958037 \\
\hline $\mathrm{C}$ & 4.498004 & -3.191673 & 0.860095 \\
\hline $\mathrm{C}$ & 5.222249 & -2.295674 & 0.081560 \\
\hline $\mathrm{H}$ & 4.848714 & -4.113520 & 1.293471 \\
\hline $\mathrm{H}$ & 6.247205 & -2.316308 & -0.249943 \\
\hline $\mathrm{N}$ & 3.160767 & 1.473672 & 0.281772 \\
\hline $\mathrm{C}$ & 3.208051 & 2.631577 & 0.958037 \\
\hline $\mathrm{C}$ & 4.498004 & 3.191673 & 0.860095 \\
\hline $\mathrm{C}$ & 5.222249 & 2.295674 & 0.081560 \\
\hline $\mathrm{H}$ & 4.848714 & 4.113520 & 1.293471 \\
\hline $\mathrm{H}$ & 6.247205 & 2.316308 & -0.249943 \\
\hline $\mathrm{N}$ & -3.160767 & -1.473672 & -0.281772 \\
\hline $\mathrm{C}$ & -3.208051 & -2.631577 & -0.958037 \\
\hline $\mathrm{C}$ & -4.498004 & -3.191673 & -0.860095 \\
\hline $\mathrm{C}$ & -5.222249 & -2.295674 & -0.081560 \\
\hline $\mathrm{H}$ & -4.848714 & -4.113520 & -1.293471 \\
\hline $\mathrm{H}$ & -6.247205 & -2.316308 & 0.249943 \\
\hline $\mathrm{N}$ & -3.160767 & 1.473672 & -0.281772 \\
\hline $\mathrm{C}$ & -3.208051 & 2.631577 & -0.958037 \\
\hline $\mathrm{C}$ & -4.498004 & 3.191673 & -0.860095 \\
\hline $\mathrm{C}$ & -5.222249 & 2.295674 & -0.081560 \\
\hline $\mathrm{H}$ & -4.848714 & 4.113520 & -1.293471 \\
\hline $\mathrm{H}$ & -6.247205 & 2.316308 & 0.249943 \\
\hline $\mathrm{C}$ & 0.460941 & 0.000000 & -3.708393 \\
\hline $\mathrm{C}$ & -0.460941 & 0.000000 & 3.708393 \\
\hline $\mathrm{H}$ & 2.333790 & -3.000310 & 1.471729 \\
\hline $\mathrm{H}$ & 2.333790 & 3.000310 & 1.471729 \\
\hline $\mathrm{H}$ & -2.333790 & -3.000310 & -1.471729 \\
\hline $\mathrm{H}$ & -2.333790 & 3.000310 & -1.471729 \\
\hline $\mathrm{F}$ & -0.162720 & 1.120852 & -3.156898 \\
\hline $\mathrm{F}$ & 0.131406 & -0.006114 & -5.065147 \\
\hline $\mathrm{F}$ & -0.164334 & -1.114739 & -3.146432 \\
\hline $\mathrm{F}$ & 0.162720 & -1.120852 & 3.156898 \\
\hline $\mathrm{F}$ & 0.164334 & 1.114739 & 3.146432 \\
\hline $\mathrm{F}$ & -0.131406 & 0.006114 & 5.065147 \\
\hline
\end{tabular}




$$
\mathbf{1 a}-\left[\mathrm{Cu}_{2}(\square-\mathrm{O})_{2}\left(\mathrm{Tp}^{b s-3 C F_{3}}\right)_{2}\right]
$$

\begin{tabular}{|c|c|c|c|}
\hline $\mathrm{Cu}$ & 1.828374 & 0.000000 & 0.000000 \\
\hline $\mathrm{Cu}$ & -1.828374 & 0.000000 & 0.000000 \\
\hline $\mathrm{O}$ & 0.000000 & 0.699180 & 0.000000 \\
\hline $\mathrm{O}$ & 0.000000 & -0.699180 & 0.000000 \\
\hline B & 4.794119 & 0.000000 & -0.861509 \\
\hline B & -4.794119 & 0.000000 & 0.861509 \\
\hline $\mathrm{H}$ & 5.953078 & 0.000000 & -1.160935 \\
\hline $\mathrm{H}$ & -5.953078 & 0.000000 & 1.160935 \\
\hline $\mathrm{N}$ & 3.940772 & 0.000000 & -2.137996 \\
\hline $\mathrm{N}$ & 4.456239 & -1.256350 & 0.011758 \\
\hline $\mathrm{N}$ & 4.456239 & 1.256350 & 0.011758 \\
\hline $\mathrm{N}$ & -3.940772 & 0.000000 & 2.137996 \\
\hline $\mathrm{N}$ & -4.456239 & -1.256350 & -0.011758 \\
\hline $\mathrm{N}$ & -4.456239 & 1.256350 & -0.011758 \\
\hline $\mathrm{N}$ & 2.575095 & 0.000000 & -2.074368 \\
\hline $\mathrm{C}$ & 2.129011 & 0.000000 & -3.335919 \\
\hline $\mathrm{C}$ & 3.212833 & 0.000000 & -4.244146 \\
\hline $\mathrm{C}$ & 4.347829 & 0.000000 & -3.447081 \\
\hline $\mathrm{H}$ & 3.169636 & 0.000000 & -5.320787 \\
\hline $\mathrm{H}$ & 5.394244 & 0.000000 & -3.704982 \\
\hline $\mathrm{N}$ & -2.575095 & 0.000000 & 2.074368 \\
\hline $\mathrm{C}$ & -2.129011 & 0.000000 & 3.335919 \\
\hline C & -3.212833 & 0.000000 & 4.244146 \\
\hline $\mathrm{C}$ & -4.347829 & 0.000000 & 3.447081 \\
\hline $\mathrm{H}$ & -3.169636 & 0.000000 & 5.320787 \\
\hline $\mathrm{H}$ & -5.394244 & 0.000000 & 3.704982 \\
\hline $\mathrm{N}$ & 3.197493 & -1.464679 & 0.467296 \\
\hline $\mathrm{C}$ & 3.223336 & -2.605418 & 1.174266 \\
\hline $\mathrm{C}$ & 4.519010 & -3.154629 & 1.183103 \\
\hline $\mathrm{C}$ & 5.276480 & -2.265295 & 0.431037 \\
\hline $\mathrm{H}$ & 4.841210 & -4.060630 & 1.666654 \\
\hline $\mathrm{H}$ & 6.320726 & -2.276719 & 0.168395 \\
\hline $\mathrm{N}$ & 3.197493 & 1.464679 & 0.467296 \\
\hline $\mathrm{C}$ & 3.223336 & 2.605418 & 1.174266 \\
\hline C & 4.519010 & 3.154629 & 1.183103 \\
\hline C & 5.276480 & 2.265295 & 0.431037 \\
\hline $\mathrm{H}$ & 4.841210 & 4.060630 & 1.666654 \\
\hline $\mathrm{H}$ & 6.320726 & 2.276719 & 0.168395 \\
\hline $\mathrm{N}$ & -3.197493 & -1.464679 & -0.467296 \\
\hline C & -3.223336 & -2.605418 & -1.174266 \\
\hline $\mathrm{C}$ & -4.519010 & -3.154629 & -1.183103 \\
\hline $\mathrm{C}$ & -5.276480 & -2.265295 & -0.431037 \\
\hline $\mathrm{H}$ & -4.841210 & -4.060630 & -1.666654 \\
\hline $\mathrm{H}$ & -6.320726 & -2.276719 & -0.168395 \\
\hline $\mathrm{N}$ & -3.197493 & 1.464679 & -0.467296 \\
\hline $\mathrm{C}$ & -3.223336 & 2.605418 & -1.174266 \\
\hline $\mathrm{C}$ & -4.519010 & 3.154629 & -1.183103 \\
\hline C & -5.276480 & 2.265295 & -0.431037 \\
\hline $\mathrm{H}$ & -4.841210 & 4.060630 & -1.666654 \\
\hline $\mathrm{H}$ & -6.320726 & 2.276719 & -0.168395 \\
\hline $\mathrm{H}$ & 1.068743 & 0.000000 & -3.537904 \\
\hline $\mathrm{H}$ & -1.068743 & 0.000000 & 3.537904 \\
\hline $\mathrm{C}$ & 1.997252 & -3.126385 & 1.816952 \\
\hline C & 1.997252 & 3.126385 & 1.816952 \\
\hline C & -1.997252 & -3.126385 & -1.816952 \\
\hline $\mathrm{C}$ & -1.997252 & 3.126385 & -1.816952 \\
\hline $\mathrm{F}$ & 1.335533 & -2.142479 & 2.547022 \\
\hline $\mathrm{F}$ & 1.089598 & -3.617544 & 0.882139 \\
\hline $\mathrm{F}$ & 2.275470 & -4.167747 & 2.698487 \\
\hline $\mathrm{F}$ & 1.335533 & 2.142479 & 2.547022 \\
\hline $\mathrm{F}$ & 2.275470 & 4.167747 & 2.698487 \\
\hline $\mathrm{F}$ & 1.089598 & 3.617544 & 0.882139 \\
\hline $\mathrm{F}$ & -1.335533 & -2.142479 & -2.547022 \\
\hline $\mathrm{F}$ & -1.089598 & -3.617544 & -0.882139 \\
\hline
\end{tabular}




$\begin{array}{llrl}F & -2.275470 & -4.167747 & -2.698487 \\ F & -1.335533 & 2.142479 & -2.547022 \\ F & -2.275470 & 4.167747 & -2.698487 \\ F & -1.089598 & 3.617544 & -0.882139\end{array}$


1b- $\left[\mathrm{Cu}_{2}(\square-\mathrm{O})_{2}(\mathrm{Tp})_{2}\right]$

\begin{tabular}{|c|c|c|c|}
\hline $\mathrm{Cu}$ & 1.413784 & 0.000000 & 0.000000 \\
\hline $\mathrm{Cu}$ & -1.413784 & 0.000000 & 0.000000 \\
\hline 0 & 0.000000 & 1.127556 & 0.000000 \\
\hline 0 & 0.000000 & -1.127556 & 0.000000 \\
\hline B & 4.524524 & 0.000000 & -0.695527 \\
\hline B & -4.524524 & 0.000000 & 0.695527 \\
\hline $\mathrm{H}$ & 5.711747 & 0.000000 & -0.857888 \\
\hline $\mathrm{H}$ & -5.711747 & 0.000000 & 0.857888 \\
\hline $\mathrm{N}$ & 3.850490 & 0.000000 & -2.084458 \\
\hline $\mathrm{N}$ & 4.051959 & -1.251358 & 0.102016 \\
\hline $\mathrm{N}$ & 4.051959 & 1.251358 & 0.102016 \\
\hline $\mathrm{N}$ & -3.850490 & 0.000000 & 2.084458 \\
\hline $\mathrm{N}$ & -4.051959 & -1.251358 & -0.102016 \\
\hline $\mathrm{N}$ & -4.051959 & 1.251358 & -0.102016 \\
\hline $\mathrm{N}$ & 2.490232 & 0.000000 & -2.211665 \\
\hline $\mathrm{C}$ & 2.236372 & 0.000000 & -3.526136 \\
\hline $\mathrm{C}$ & 3.437712 & 0.000000 & -4.273683 \\
\hline $\mathrm{C}$ & 4.444874 & 0.000000 & -3.320003 \\
\hline $\mathrm{H}$ & 3.550002 & 0.000000 & -5.345732 \\
\hline $\mathrm{H}$ & 5.517863 & 0.000000 & -3.422348 \\
\hline $\mathrm{N}$ & -2.490232 & 0.000000 & 2.211665 \\
\hline $\mathrm{C}$ & -2.236372 & 0.000000 & 3.526136 \\
\hline $\mathrm{C}$ & -3.437712 & 0.000000 & 4.273683 \\
\hline $\mathrm{C}$ & -4.444874 & 0.000000 & 3.320003 \\
\hline $\mathrm{H}$ & -3.550002 & 0.000000 & 5.345732 \\
\hline $\mathrm{H}$ & -5.517863 & 0.000000 & 3.422348 \\
\hline $\mathrm{N}$ & 2.732265 & -1.420838 & 0.381757 \\
\hline $\mathrm{C}$ & 2.591104 & -2.564163 & 1.070703 \\
\hline $\mathrm{C}$ & 3.855265 & -3.159340 & 1.246413 \\
\hline $\mathrm{C}$ & 4.753865 & -2.298916 & 0.619576 \\
\hline $\mathrm{H}$ & 4.080851 & -4.081474 & 1.755653 \\
\hline $\mathrm{H}$ & 5.823791 & -2.355107 & 0.506167 \\
\hline $\mathrm{N}$ & 2.732265 & 1.420838 & 0.381757 \\
\hline $\mathrm{C}$ & 2.591104 & 2.564163 & 1.070703 \\
\hline $\mathrm{C}$ & 3.855265 & 3.159340 & 1.246413 \\
\hline $\mathrm{C}$ & 4.753865 & 2.298916 & 0.619576 \\
\hline $\mathrm{H}$ & 4.080851 & 4.081474 & 1.755653 \\
\hline $\mathrm{H}$ & 5.823791 & 2.355107 & 0.506167 \\
\hline $\mathrm{N}$ & -2.732265 & -1.420838 & -0.381757 \\
\hline $\mathrm{C}$ & -2.591104 & -2.564163 & -1.070703 \\
\hline $\mathrm{C}$ & -3.855265 & -3.159340 & -1.246413 \\
\hline $\mathrm{C}$ & -4.753865 & -2.298916 & -0.619576 \\
\hline $\mathrm{H}$ & -4.080851 & -4.081474 & -1.755653 \\
\hline $\mathrm{H}$ & -5.823791 & -2.355107 & -0.506167 \\
\hline $\mathrm{N}$ & -2.732265 & 1.420838 & -0.381757 \\
\hline $\mathrm{C}$ & -2.591104 & 2.564163 & -1.070703 \\
\hline $\mathrm{C}$ & -3.855265 & 3.159340 & -1.246413 \\
\hline $\mathrm{C}$ & -4.753865 & 2.298916 & -0.619576 \\
\hline $\mathrm{H}$ & -4.080851 & 4.081474 & -1.755653 \\
\hline $\mathrm{H}$ & -5.823791 & 2.355107 & -0.506167 \\
\hline $\mathrm{H}$ & 1.217315 & 0.000000 & -3.883476 \\
\hline $\mathrm{H}$ & -1.217315 & 0.000000 & 3.883476 \\
\hline $\mathrm{H}$ & 1.613550 & -2.886091 & 1.392943 \\
\hline $\mathrm{H}$ & 1.613550 & 2.886091 & 1.392943 \\
\hline $\mathrm{H}$ & -1.613550 & -2.886091 & -1.392943 \\
\hline $\mathrm{H}$ & -1.613550 & 2.886091 & -1.392943 \\
\hline
\end{tabular}


1b- $\left[\mathrm{Cu}_{2}(\square-\mathrm{O})_{2}\left(\mathrm{Tp}^{5 F}\right)_{2}\right]$

\begin{tabular}{|c|c|c|c|}
\hline $\mathrm{Cu}$ & 1.413184 & 0.000000 & 0.000000 \\
\hline $\mathrm{Cu}$ & -1.413184 & 0.000000 & 0.000000 \\
\hline 0 & 0.000000 & 1.126968 & 0.000000 \\
\hline 0 & 0.000000 & -1.126968 & 0.000000 \\
\hline B & 4.536165 & 0.000000 & -0.721036 \\
\hline B & -4.536165 & 0.000000 & 0.721036 \\
\hline $\mathrm{H}$ & 5.711484 & 0.000000 & -0.890600 \\
\hline $\mathrm{H}$ & -5.711484 & 0.000000 & 0.890600 \\
\hline $\mathrm{N}$ & 3.833058 & 0.000000 & -2.096387 \\
\hline $\mathrm{N}$ & 4.054269 & -1.247380 & 0.080372 \\
\hline $\mathrm{N}$ & 4.054269 & 1.247380 & 0.080372 \\
\hline $\mathrm{N}$ & -3.833058 & 0.000000 & 2.096387 \\
\hline $\mathrm{N}$ & -4.054269 & -1.247380 & -0.080372 \\
\hline $\mathrm{N}$ & -4.054269 & 1.247380 & -0.080372 \\
\hline $\mathrm{N}$ & 2.462879 & 0.000000 & -2.212029 \\
\hline $\mathrm{C}$ & 2.193069 & 0.000000 & -3.522367 \\
\hline $\mathrm{C}$ & 3.376987 & 0.000000 & -4.298503 \\
\hline $\mathrm{C}$ & 4.375144 & 0.000000 & -3.346290 \\
\hline $\mathrm{H}$ & 3.487659 & 0.000000 & -5.368562 \\
\hline $\mathrm{F}$ & 5.733392 & 0.000000 & -3.517498 \\
\hline $\mathrm{N}$ & -2.462879 & 0.000000 & 2.212029 \\
\hline $\mathrm{C}$ & -2.193069 & 0.000000 & 3.522367 \\
\hline $\mathrm{C}$ & -3.376987 & 0.000000 & 4.298503 \\
\hline $\mathrm{C}$ & -4.375144 & 0.000000 & 3.346290 \\
\hline $\mathrm{H}$ & -3.487659 & 0.000000 & 5.368562 \\
\hline $\mathrm{F}$ & -5.733392 & 0.000000 & 3.517498 \\
\hline $\mathrm{N}$ & 2.730870 & -1.422830 & 0.374244 \\
\hline $\mathrm{C}$ & 2.597122 & -2.565662 & 1.064082 \\
\hline $\mathrm{C}$ & 3.857159 & -3.171162 & 1.237564 \\
\hline $\mathrm{C}$ & 4.729070 & -2.301649 & 0.601597 \\
\hline $\mathrm{H}$ & 4.098374 & -4.089962 & 1.741858 \\
\hline $\mathrm{F}$ & 6.081262 & -2.393117 & 0.460183 \\
\hline $\mathrm{N}$ & 2.730870 & 1.422830 & 0.374244 \\
\hline $\mathrm{C}$ & 2.597122 & 2.565662 & 1.064082 \\
\hline $\mathrm{C}$ & 3.857159 & 3.171162 & 1.237564 \\
\hline $\mathrm{C}$ & 4.729070 & 2.301649 & 0.601597 \\
\hline $\mathrm{H}$ & 4.098374 & 4.089962 & 1.741858 \\
\hline $\mathrm{F}$ & 6.081262 & 2.393117 & 0.460183 \\
\hline $\mathrm{N}$ & -2.730870 & -1.422830 & -0.374244 \\
\hline $\mathrm{C}$ & -2.597122 & -2.565662 & -1.064082 \\
\hline $\mathrm{C}$ & -3.857159 & -3.171162 & -1.237564 \\
\hline $\mathrm{C}$ & -4.729070 & -2.301649 & -0.601597 \\
\hline $\mathrm{H}$ & -4.098374 & -4.089962 & -1.741858 \\
\hline $\mathrm{F}$ & -6.081262 & -2.393117 & -0.460183 \\
\hline $\mathrm{N}$ & -2.730870 & 1.422830 & -0.374244 \\
\hline $\mathrm{C}$ & -2.597122 & 2.565662 & -1.064082 \\
\hline $\mathrm{C}$ & -3.857159 & 3.171162 & -1.237564 \\
\hline $\mathrm{C}$ & -4.729070 & 2.301649 & -0.601597 \\
\hline $\mathrm{H}$ & -4.098374 & 4.089962 & -1.741858 \\
\hline $\mathrm{F}$ & -6.081262 & 2.393117 & -0.460183 \\
\hline $\mathrm{H}$ & 1.169837 & 0.000000 & -3.866409 \\
\hline $\mathrm{H}$ & -1.169837 & 0.000000 & 3.866409 \\
\hline $\mathrm{H}$ & 1.623089 & -2.889557 & 1.394648 \\
\hline $\mathrm{H}$ & 1.623089 & 2.889557 & 1.394648 \\
\hline $\mathrm{H}$ & -1.623089 & -2.889557 & -1.394648 \\
\hline $\mathrm{H}$ & -1.623089 & 2.889557 & -1.394648 \\
\hline
\end{tabular}




$$
\mathbf{1 b}-\left[\mathrm{Cu}_{2}(\square-\mathrm{O})_{2}\left(\mathrm{Tp}^{3 \mathrm{CH}_{3}}\right)_{2}\right]
$$

\begin{tabular}{|c|c|c|c|}
\hline $\mathrm{Cu}$ & 1.483005 & 0.000000 & 0.000000 \\
\hline $\mathrm{Cu}$ & -1.483005 & 0.000000 & 0.000000 \\
\hline 0 & 0.000000 & 1.058466 & 0.000000 \\
\hline 0 & 0.000000 & -1.058466 & 0.000000 \\
\hline B & 4.453772 & 0.000000 & -1.030039 \\
\hline B & -4.453772 & 0.000000 & 1.030039 \\
\hline $\mathrm{H}$ & 5.605010 & 0.000000 & -1.366684 \\
\hline $\mathrm{H}$ & -5.605010 & 0.000000 & 1.366684 \\
\hline $\mathrm{N}$ & 3.604634 & 0.000000 & -2.316422 \\
\hline $\mathrm{N}$ & 4.112536 & -1.245587 & -0.166294 \\
\hline $\mathrm{N}$ & 4.112536 & 1.245587 & -0.166294 \\
\hline $\mathrm{N}$ & -3.604634 & 0.000000 & 2.316422 \\
\hline $\mathrm{N}$ & -4.112536 & -1.245587 & 0.166294 \\
\hline $\mathrm{N}$ & -4.112536 & 1.245587 & 0.166294 \\
\hline $\mathrm{N}$ & 2.239806 & 0.000000 & -2.265124 \\
\hline $\mathrm{C}$ & 1.800338 & 0.000000 & -3.533494 \\
\hline $\mathrm{C}$ & 2.903618 & 0.000000 & -4.428091 \\
\hline C & 4.028087 & 0.000000 & -3.618650 \\
\hline $\mathrm{H}$ & 2.872545 & 0.000000 & -5.506128 \\
\hline $\mathrm{H}$ & 5.077836 & 0.000000 & -3.863778 \\
\hline $\mathrm{N}$ & -2.239806 & 0.000000 & 2.265124 \\
\hline $\mathrm{C}$ & -1.800338 & 0.000000 & 3.533494 \\
\hline $\mathrm{C}$ & -2.903618 & 0.000000 & 4.428091 \\
\hline $\mathrm{C}$ & -4.028087 & 0.000000 & 3.618650 \\
\hline $\mathrm{H}$ & -2.872545 & 0.000000 & 5.506128 \\
\hline $\mathrm{H}$ & -5.077836 & 0.000000 & 3.863778 \\
\hline $\mathrm{N}$ & 2.851539 & -1.441738 & 0.322752 \\
\hline $\mathrm{C}$ & 2.849348 & -2.593244 & 1.029651 \\
\hline C & 4.149192 & -3.149036 & 0.990437 \\
\hline C & 4.912116 & -2.272879 & 0.229965 \\
\hline $\mathrm{H}$ & 4.473593 & -4.064820 & 1.457257 \\
\hline $\mathrm{H}$ & 5.950722 & -2.302844 & -0.055365 \\
\hline $\mathrm{N}$ & 2.851539 & 1.441738 & 0.322752 \\
\hline C & 2.849348 & 2.593244 & 1.029651 \\
\hline $\mathrm{C}$ & 4.149192 & 3.149036 & 0.990437 \\
\hline C & 4.912116 & 2.272879 & 0.229965 \\
\hline $\mathrm{H}$ & 4.473593 & 4.064820 & 1.457257 \\
\hline $\mathrm{H}$ & 5.950722 & 2.302844 & -0.055365 \\
\hline $\mathrm{N}$ & -2.851539 & -1.441738 & -0.322752 \\
\hline $\mathrm{C}$ & -2.849348 & -2.593244 & -1.029651 \\
\hline C & -4.149192 & -3.149036 & -0.990437 \\
\hline $\mathrm{C}$ & -4.912116 & -2.272879 & -0.229965 \\
\hline $\mathrm{H}$ & -4.473593 & -4.064820 & -1.457257 \\
\hline $\mathrm{H}$ & -5.950722 & -2.302844 & 0.055365 \\
\hline $\mathrm{N}$ & -2.851539 & 1.441738 & -0.322752 \\
\hline $\mathrm{C}$ & -2.849348 & 2.593244 & -1.029651 \\
\hline $\mathrm{C}$ & -4.149192 & 3.149036 & -0.990437 \\
\hline C & -4.912116 & 2.272879 & -0.229965 \\
\hline $\mathrm{H}$ & -4.473593 & 4.064820 & -1.457257 \\
\hline $\mathrm{H}$ & -5.950722 & 2.302844 & 0.055365 \\
\hline $\mathrm{C}$ & 0.335764 & 0.000000 & -3.853099 \\
\hline $\mathrm{C}$ & -0.335764 & 0.000000 & 3.853099 \\
\hline $\mathrm{C}$ & 1.629894 & -3.129871 & 1.715374 \\
\hline $\mathrm{C}$ & 1.629894 & 3.129871 & 1.715374 \\
\hline C & -1.629894 & -3.129871 & -1.715374 \\
\hline $\mathrm{C}$ & -1.629894 & 3.129871 & -1.715374 \\
\hline $\mathrm{H}$ & -0.266545 & -0.000012 & -2.937834 \\
\hline $\mathrm{H}$ & 0.060502 & 0.885636 & -4.436509 \\
\hline $\mathrm{H}$ & 0.060507 & -0.885623 & -4.436530 \\
\hline $\mathrm{H}$ & 0.266545 & 0.000012 & 2.937834 \\
\hline $\mathrm{H}$ & -0.060507 & 0.885623 & 4.436530 \\
\hline $\mathrm{H}$ & -0.060502 & -0.885636 & 4.436509 \\
\hline $\mathrm{H}$ & 0.746393 & -2.534553 & 1.462088 \\
\hline $\mathrm{H}$ & 1.438290 & -4.165650 & 1.416073 \\
\hline
\end{tabular}




$\begin{array}{rrrr}\mathrm{H} & 1.753051 & -3.108319 & 2.803262 \\ \mathrm{H} & 0.746393 & 2.534553 & 1.462088 \\ \mathrm{H} & 1.753051 & 3.108319 & 2.803262 \\ \mathrm{H} & 1.438290 & 4.165650 & 1.416073 \\ \mathrm{H} & -0.746393 & -2.534553 & -1.462088 \\ \mathrm{H} & -1.438290 & -4.165650 & -1.416073 \\ \mathrm{H} & -1.753051 & -3.108319 & -2.803262 \\ \mathrm{H} & -0.746393 & 2.534553 & -1.462088 \\ \mathrm{H} & -1.753051 & 3.108319 & -2.803262 \\ \mathrm{H} & -1.438290 & 4.165650 & -1.416073\end{array}$


1b- $\left[\mathrm{Cu}_{2}(\square-\mathrm{O})_{2}\left(\mathrm{Tp}^{a p-3 C F_{3}}\right)_{2}\right]$

\begin{tabular}{|c|c|c|c|}
\hline $\mathrm{Cu}$ & 1.406060 & 0.000000 & 0.000000 \\
\hline $\mathrm{Cu}$ & -1.406060 & 0.000000 & 0.000000 \\
\hline 0 & 0.000000 & 1.127646 & 0.000000 \\
\hline 0 & 0.000000 & -1.127646 & 0.000000 \\
\hline B & 4.427565 & 0.000000 & -1.012693 \\
\hline B & -4.427565 & 0.000000 & 1.012693 \\
\hline $\mathrm{H}$ & 5.586028 & 0.000000 & -1.314922 \\
\hline $\mathrm{H}$ & -5.586028 & 0.000000 & 1.314922 \\
\hline $\mathrm{N}$ & 3.608813 & 0.000000 & -2.341707 \\
\hline $\mathrm{N}$ & 4.036310 & -1.250501 & -0.181110 \\
\hline $\mathrm{N}$ & 4.036310 & 1.250501 & -0.181110 \\
\hline $\mathrm{N}$ & -3.608813 & 0.000000 & 2.341707 \\
\hline $\mathrm{N}$ & -4.036310 & -1.250501 & 0.181110 \\
\hline $\mathrm{N}$ & -4.036310 & 1.250501 & 0.181110 \\
\hline $\mathrm{N}$ & 2.258603 & 0.000000 & -2.317664 \\
\hline C & 1.863801 & 0.000000 & -3.596566 \\
\hline $\mathrm{C}$ & 2.969482 & 0.000000 & -4.476912 \\
\hline $\mathrm{C}$ & 4.070832 & 0.000000 & -3.634739 \\
\hline $\mathrm{H}$ & 2.953686 & 0.000000 & -5.553411 \\
\hline $\mathrm{H}$ & 5.125761 & 0.000000 & -3.852258 \\
\hline $\mathrm{N}$ & -2.258603 & 0.000000 & 2.317664 \\
\hline $\mathrm{C}$ & -1.863801 & 0.000000 & 3.596566 \\
\hline C & -2.969482 & 0.000000 & 4.476912 \\
\hline C & -4.070832 & 0.000000 & 3.634739 \\
\hline $\mathrm{H}$ & -2.953686 & 0.000000 & 5.553411 \\
\hline $\mathrm{H}$ & -5.125761 & 0.000000 & 3.852258 \\
\hline $\mathrm{N}$ & 2.754050 & -1.413071 & 0.248256 \\
\hline $\mathrm{C}$ & 2.682962 & -2.561686 & 0.936873 \\
\hline $\mathrm{C}$ & 3.954034 & -3.169056 & 0.959590 \\
\hline $\mathrm{C}$ & 4.782993 & -2.310835 & 0.242661 \\
\hline $\mathrm{H}$ & 4.227346 & -4.098491 & 1.430494 \\
\hline $\mathrm{H}$ & 5.832277 & -2.375706 & 0.006760 \\
\hline $\mathrm{N}$ & 2.754050 & 1.413071 & 0.248256 \\
\hline $\mathrm{C}$ & 2.682962 & 2.561686 & 0.936873 \\
\hline C & 3.954034 & 3.169056 & 0.959590 \\
\hline $\mathrm{C}$ & 4.782993 & 2.310835 & 0.242661 \\
\hline $\mathrm{H}$ & 4.227346 & 4.098491 & 1.430494 \\
\hline $\mathrm{H}$ & 5.832277 & 2.375706 & 0.006760 \\
\hline $\mathrm{N}$ & -2.754050 & -1.413071 & -0.248256 \\
\hline $\mathrm{C}$ & -2.682962 & -2.561686 & -0.936873 \\
\hline $\mathrm{C}$ & -3.954034 & -3.169056 & -0.959590 \\
\hline $\mathrm{C}$ & -4.782993 & -2.310835 & -0.242661 \\
\hline $\mathrm{H}$ & -4.227346 & -4.098491 & -1.430494 \\
\hline $\mathrm{H}$ & -5.832277 & -2.375706 & -0.006760 \\
\hline $\mathrm{N}$ & -2.754050 & 1.413071 & -0.248256 \\
\hline $\mathrm{C}$ & -2.682962 & 2.561686 & -0.936873 \\
\hline C & -3.954034 & 3.169056 & -0.959590 \\
\hline $\mathrm{C}$ & -4.782993 & 2.310835 & -0.242661 \\
\hline $\mathrm{H}$ & -4.227346 & 4.098491 & -1.430494 \\
\hline $\mathrm{H}$ & -5.832277 & 2.375706 & -0.006760 \\
\hline C & 0.425208 & 0.000000 & -3.928159 \\
\hline $\mathrm{C}$ & -0.425208 & 0.000000 & 3.928159 \\
\hline $\mathrm{H}$ & 1.746007 & -2.878876 & 1.366354 \\
\hline $\mathrm{H}$ & 1.746007 & 2.878876 & 1.366354 \\
\hline $\mathrm{H}$ & -1.746007 & -2.878876 & -1.366354 \\
\hline $\mathrm{H}$ & -1.746007 & 2.878876 & -1.366354 \\
\hline$F$ & -0.239714 & 1.116850 & -3.417647 \\
\hline $\mathrm{F}$ & 0.195452 & 0.001734 & -5.305587 \\
\hline $\mathrm{F}$ & -0.239040 & -1.118583 & -3.420573 \\
\hline $\mathrm{F}$ & 0.239714 & -1.116850 & 3.417647 \\
\hline $\mathrm{F}$ & 0.239040 & 1.118583 & 3.420573 \\
\hline $\mathrm{F}$ & -0.195452 & -0.001734 & 5.305587 \\
\hline
\end{tabular}

